\title{
Initial simulations spanning the upper convection zone to the corona
}

\author{
Viggo H. Hansteen ${ }^{1} \dagger$ \\ ${ }^{1}$ Institute of Theoretical Astrophysics, University of Oslo, Norway
}

\begin{abstract}
A major goal in solar physics has during the last five decades been to find how energy flux generated in the solar convection zone is transported and dissipated in the outer solar layers. Progress in this field has been slow and painstaking. However, advances in computer hardware and numerical methods, vastly increased observational capababilities and growing physical insight seem finally to be leading towards understanding. Here we present exploratory numerical MHD models that span the entire solar atmosphere from the upper convection zone to the lower corona. These models include non-grey, non-lte radiative transport in the photosphere and chromosphere, optically thin radiative losses as well as magnetic field-aligned heat conduction in the transition region and corona.
\end{abstract}

\section{Current Models}

The figures shown below give examples of simulations that are being run to highlight the physical processes occuring as a result of the interplay between the solar convection zone and photosphere and the chromosphere and corona.

We solve the basic equations of MHD using the method described in detail by Galsgaard and Nordlund at http://www.astro.ku.dk/ kg. Radiative losses from the photosphere by solving the transport equation as descibed by Skartlien (2000). Thermal conduction along the magnetic field in the corona and transition region is treated by operator splitting and using a multi-grid solver.

The models shown here are convectively unstable due the radiative losses in the photosphere. The average temperature at the bottom boundary is maintained by setting the entropy of the fluid entering through the bottom boundary. The bottom boundary, based on characteristic extropolation, is otherwise open, allowing fluid to enter and leave the computational domain as required. The upper boundary in both models is maintained by requiring the temperature to tend towards $1 \mathrm{MK}$ on a timescale of $10 \mathrm{~s}$. The magnetic field is simply extrapolated while the other hydrodynamic variables are set using extropolated characteristics. The horizontal boundaries are periodic.

The model shown is run on a grid of $64 \times 32 \times 150$ points spanning $8 \times 4 \times 12 \mathrm{Mm}^{3}$. This model was initially based on a convectively relaxed $2 \mathrm{~d}$ model expanded in the $y$-direction. At the start of the simulation the model was given a uniform vertical magnetic field of $B_{z}=10$ Gauss, a horizontal field of $B_{x}=10$ Gauss is introduced at the bottom boundary in inflow regions. The model has currently run roughly 15 minutes of solar time, as may be seen from the incomplete relaxation in the $y$-direction in the temperature structure at the bottom boundary as seen in figure 2 .

The models presented here show that it is now indeed feasible to study the outer layers of the sun numerically as one complete system.

$\dagger$ Also at Center of Mathematics for Applications, University of Oslo, P.O. Box 1053 Blindern, N-0316 Oslo, Norway. 

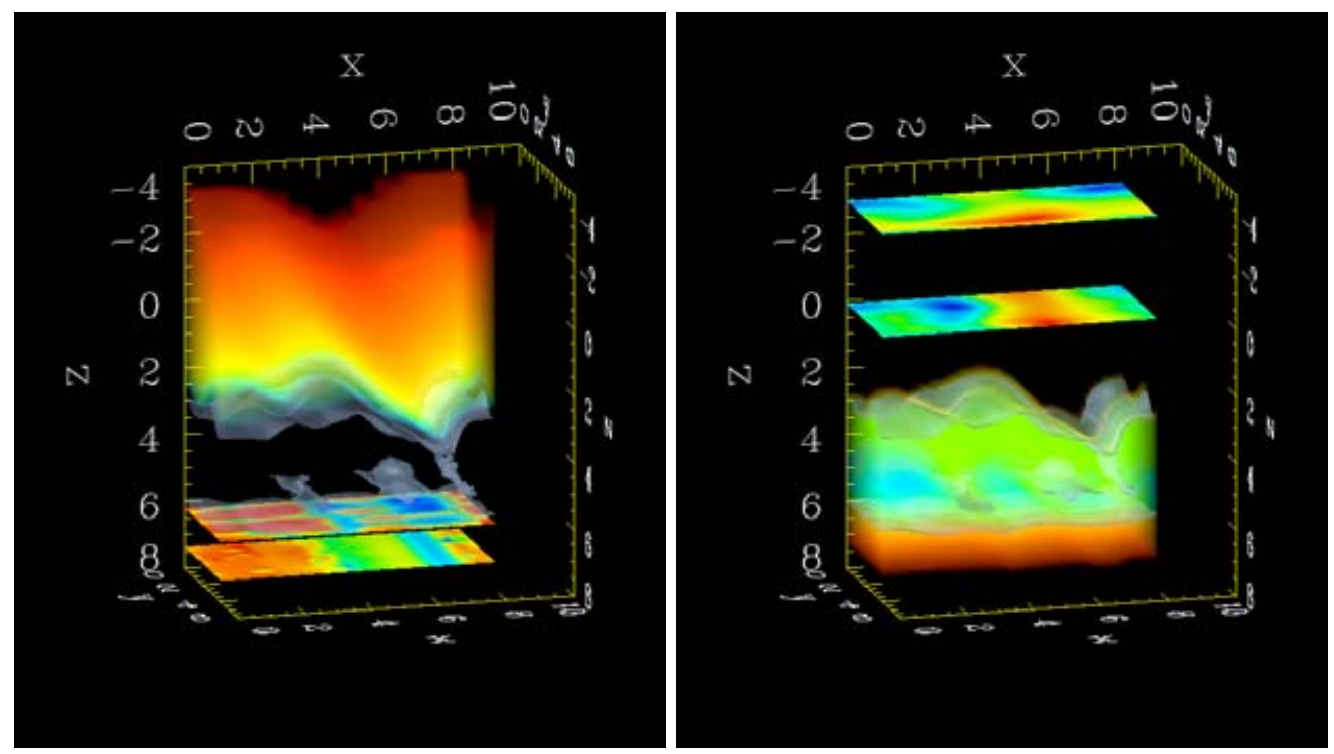

Figure 1. The temperature structure in the corona is shown in the left panel and in the chromosphere in the right panel by the colored regions. The isosurfaces are set to pick out regions where $T_{g}=7000 \mathrm{~K}$, thus deliniating the chromosphere from the corona and photosphere/convection region.
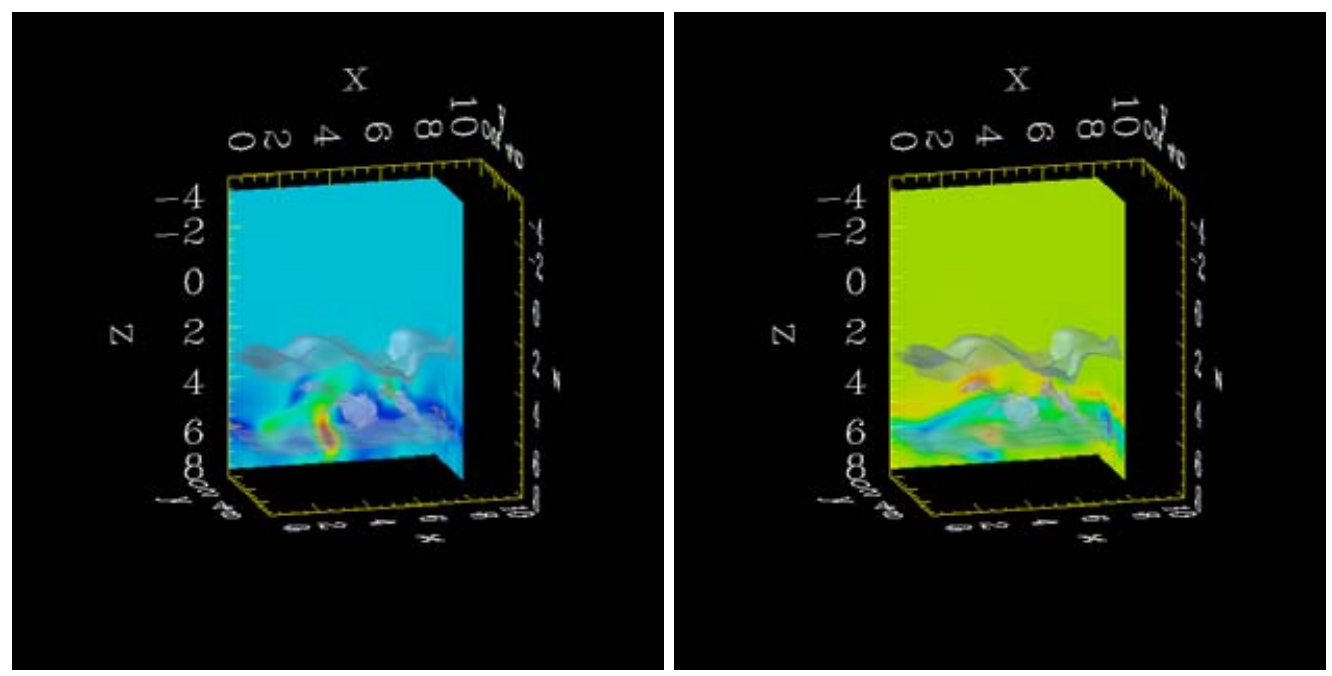

Figure 2. The left hand panel we show the vertical magnetic field $B_{z}$ along two slabs, in the right panel the horizontal component $B_{x}$ is shown. The isosurfaces are set $T_{g}=7000 \mathrm{~K}$.

\section{Acknowledgements}

This research was supported by the European Community's Human Potential Programme through the European Solar Magnetism Network (TOSTISP, contract HPRNCT-2001-00310), by grant 146467/420 from the Norwegian Research Council.

\section{References}

Skartlien, R. 2000, ApJ, 536, 465. 